\title{
12 Erste Erfahrungen im Rahmen des Projektes eMeldewesen.nrw - identifizierte Problemlagen und lösungsansätze
}

\author{
Lars Treinat
}

\subsection{Hintergrund}

Angesichts der in den letzten Jahren immer wieder auftretenden Erkrankungswellen im Zusammenhang mit Erregern wie $\mathrm{H}_{5} \mathrm{~N}_{1}, \mathrm{H}_{5} \mathrm{~N}_{9}$, Coronavirus, $\mathrm{H} 1 \mathrm{~N} 1$ oder EHEC, denen das Potential zu Pandemien zugeschrieben wird, entwickelte sich eine breitere Diskussion, ob die etablierten Meldewege noch den Anforderungen einer globalisierten Welt gewachsen sind, in der auch Krankheitserreger im Gefolge von Menschen, Tieren und Gütern immer mobiler werden. Vor diesem Hintergrund wurden im Bundesland Nordrhein-Westfalen (NRW) Überlegungen angestellt, welche Faktoren für die Eindämmung eines Ausbruchsgeschehens kritisch sind und auf welche Weise diese Faktoren beeinflusst werden können. Dabei zeigte sich, dass die zeitnahe Information der für die Einleitung von Maßnahmen vor Ort zuständigen Gesundheitsbehörden eine entscheidende Rolle spielt [LZG.NRW, 2012]. In Anbetracht der Rahmenbedingungen in NRW als Flächenland mit mehr als 17 Millionen Einwohnern von denen ein großer Teil in Ballungsräumen lebt - und einem Netz von 56 Gesundheitsämtern auf lokaler Ebene, wurde der Gedanke geboren, zunächst im Kleinen neue Wege zu erproben, um praktikable Lösungen für eine Verkürzung der Zeitspanne zwischen Erkrankung und Verfügbarkeit der Information darüber zu finden. Zu diesem Zweck beauftragte 2011 das Ministerium für Gesundheit, Emanzipation, Pflege und Alter des Landes Nordrhein-Westfalen (MCEPA) das Zentrum für Telematik und Telemedizin (ZTG), eine erste Mach- 
B Der fachliche Hintergrund: Terminologien und Ordnungssysteme in der Patientenversorgung, medizinischen Forschung und Gesundheitswirtschaft

barkeitserprobung für einen elektronischen Meldeweg durchzuführen. Aufbauend auf diesem ersten Pilotversuch im Rhein-Kreis Neuss wird aktuell an der Weiterentwicklung und Erprobung einer strukturierten Labormeldung gearbeitet. Im Rahmen dieses Artikels sollen die in der Folge identifizierten Problemlagen und Lösungsansätze betrachtet werden. Mit dem Blick auf eine maschinelle Verarbeitbarkeit der Meldungen spielt das Thema Semantik hierbei eine herausragende Rolle, die eingehender beleuchtet werden soll.

\subsection{Problemanalyse}

Die Meldepflichten, Fristen und Meldewege sind in Deutschland überwiegend im Infektionsschutzgesetz (IfSG) bundesrechtlich geregelt. Im Kontext der Aktivitäten in NRW stehen primär die namentlichen Meldungen nach $\mathbb{} 6$ (Arztmeldung) und $₫ 7$ Abs. 1 und 2 IfSG (Labormeldung) im Blickpunkt, welche direkt an das zuständige Gesundheitsamt auf Kreisebene übermittelt werden. Nicht explizit geregelt ist die Art, wie die Meldung an die vorgesehenen Empfänger übermittelt wird. Die Meldungen werden heute meist als Fax versendet. Dies hat zur Folge, dass der größte Teil der Meldungen durch die zuständigen Mitarbeiter aufwändig abgetippt und interpretiert werden muss, was mit einem erheblichen Zeitaufwand verbunden ist. Ein weiteres Problem ist, dass in einem nicht unerheblichen Ausmaß, insbesondere bei meldepflichtigen Erkrankungen, bei denen aufgrund des klinischen Bildes keine Labordiagnostik durchgeführt wird, eine Meldung nicht erfolgt [Mette et al., 2011]. In Abbildung 1 werden die unterschiedlichen Aspekte und Problemlagen dargestellt, die bei der Einführung eines elektronischen Meldeweges zu beachten sind.

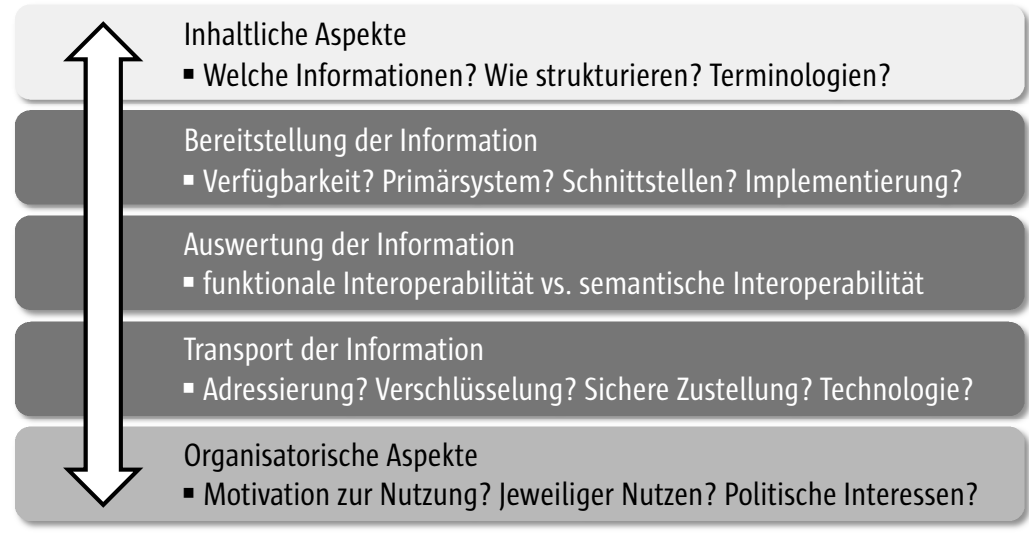

Abb. 1 Ein elektronischer Meldeweg - ein vielschichtiges Problem. ( ) ZTG GmbH, 2014

Für den sicheren Transport der als besonders schutzwürdig einzustufenden Daten (vgl. $\mathbb{S} 3_{3}$ Abs. 9 Bundesdatenschutzgesetz) gibt es aus technischer Sicht 
eine Reihe von bereits heute verfügbaren technischen Lösungen, welche die Vertraulichkeit der Übermittlung sicherstellen können. Hier bietet sich die Nutzung von sicheren Netzwerken (z.B. KV-SafeNet [KBV, 2014a], DOI [BVA, 2014], Telematikinfrastruktur [gematik, 2014b]) und darauf aufsetzenden Transportanwendungen mit Ende-zu-Ende-Verschlüsselung (z.B. D2D [D2D], KV-Connect [KV-Telematik], OSCI-Transport [KoSIT, 2014], KOM-LE [gematik, 2014a]) an.

Die organisatorischen Aspekte betreffen primär die Motivation der Beteiligten, eine elektronische Meldung einzusetzen. Es ist davon auszugehen, dass der Nutzen unter den gegebenen Rahmenbedingungen überwiegend beim Meldungsempfänger, d.h. den Gesundheitsbehörden liegt. Für einen flächendeckenden Einsatz müsste diese asymmetrische Verteilung des Nutzens durch gesetzliche Regelungen oder finanzielle Anreize ausgeglichen werden.

Bezüglich der Auswertbarkeit der elektronischen Meldungen ist die funktionale Interoperabilität (d.h. ein Datensatz wird mit einer einheitlichen Struktur und Syntax verwendet) als Mindestanforderung anzusehen. Das Potential einer elektronischen Meldung kann jedoch nur bei einer weitgehenden semantischen Interoperabilität (d.h. auch bezüglich der verwendeten Begriffe und ihrer Bedeutungen besteht ein einheitliches Verständnis) sinnvoll genutzt werden. Hinsichtlich der Frage, welche Informationen übermittelt werden sollen, stecken die gesetzlichen Vorgaben einen klaren Rahmen ab. Im Kontext des NRW-Projektes wurden sowohl für die Arztmeldung als auch die Labormeldung strukturierte Datensätze auf Basis von HL7/CDA [HL7, 2007-2014b] entwickelt, die auf der Grundlage des elektronischen Arztbriefs [HL7, 20072014a] aufbauen. Die wesentlich komplexere Frage ist, auf welche Weise eine semantische Standardisierung realisiert werden kann. Bezüglich dieser Fragen wurde bei den Aktivitäten in NRW darauf geachtet, dass eine inhaltliche Abstimmung mit dem Robert Koch-Institut (RKI) und dem DEMIS-Projekt [Fraunhofer-Wiki, 2014] gesucht wurde.

Es ist davon auszugehen, dass bei den Meldepflichtigen begrenzte Neigung besteht, mehr Aufwand zu betreiben als zur Erfüllung der gesetzlichen Meldepflicht unbedingt erforderlich ist. Die Implementierung eines elektronischen Meldeweges sollte daher darauf ausgelegt sein, bereits bei den meldepflichtigen Akteuren vorhandene Daten zu nutzen und die Erzeugung der Meldung möglichst weitgehend automatisch durchzuführen. Grundsätzlich besteht dabei die Möglichkeit, auf ggf. bereits genutzte, standardisierte Vokabulare oder in den Primärsystemen verwendete proprietäre Value-Sets zuzugreifen.

\subsection{Herausforderung Semantik}

An dieser Stelle sollen potentiell geeignete Lösungsansätze für eine Übermittlung sowie die damit verbundenen Vor- und Nachteile betrachtet werden. 
B Der fachliche Hintergrund: Terminologien und Ordnungssysteme in der

Patientenversorgung, medizinischen Forschung und Gesundheitswirtschaft

Dabei ist anzumerken, dass bei Ärzten und Krankenhäusern aufgrund externer Anforderungen standardisierte Kodierungen für Krankheiten - zumindest im Hintergrund - verwendet werden. Bei den Laboren werden, soweit bekannt, individuelle Kodierungen für Erreger, Nachweismethoden und Materialien verwendet. Dies ist bemerkenswert, da angesichts der zunehmenden Spezialisierung im Laborbereich häufig Befunde zwischen Laboren ausgetauscht werden, welche nicht selten mittels Mappingtabellen auf Kommunikationsservern in die Kodierung des empfangenden/anfordernden Labors übersetzt werden müssen.

\subsection{ICD-10}

In den Arztinformationssystemen bei niedergelassenen Ärzten wird neben umfangreichen Freitextdokumentationen zu Abrechnungszwecken auch die amtliche Klassifikation von Diagnosen auf Basis der ICD-10 GM [DIMDI, 2014] genutzt. Dies ermöglicht weitestgehend, die im IfSG aufgeführten Infektionskrankheiten anhand der entsprechenden ICD-10-Kodes zu identifizieren. Im Rahmen der Zertifizierung von Softwareprodukten, die für die Abrechnung mit den Kassenärztlichen Vereinigungen zugelassenen sind, ist die Implementierung eines Hinweismechanismus auf möglicherweise meldepflichtige Fälle vorgesehen [KBV, 2014b]. Auch bei den Krankenhäusern wird die ICD-10 GM für Zwecke der Abrechnung und Qualitätssicherung flächendeckend verwendet. Daher sind die ICD-1o-Kodierungen grundsätzlich für die Übermittlung von meldepflichtigen Erkrankungen an das Gesundheitsamt geeignet. Es ist jedoch zu beachten, dass die Kodierqualität z.T. unterschiedlich und abhängig vom primären Verwendungszweck ist. Für die Arztmeldung werden jedoch neben der Erkrankung weitere relevante Kontextinformationen (z.B. Symptome, Kontaktanamnese, Beschäftigung des Patienten im Lebensmittelbereich, Häufung von Erkrankungen) benötigt, welche die ICD-1o nicht abbilden kann. Daher werden zusätzlich weitere Value-Sets oder Freitextfelder benötigt.

\subsection{LOINC}

Bei der Suche nach einer geeigneten Terminologie zur Abbildung von Erregern, Nachweismethoden und Materialien für eine elektronische Labormeldung wurde als ein potentieller Kandidat LOINC (Logical Observation Identifiers Names and Codes) [Regenstrief-Institute, 1994-2014] ins Auge gefasst. Im Rahmen des aktuellen Projektes in NRW wurde durch ein Team der Hochschule Niederrhein unter Leitung von Prof. Dr. Sylvia Thun ein Abgleich der in Deutschland nach IfSG meldepflichtigen Erreger und der zur Verfügung stehenden LOINC-Kodes durchgeführt, wobei auch die Falldefinitionen des RKI [RKI, 2013] hinsichtlich Material und Nachweismethode berücksichtigt 
wurden. Vorteilhaft an diesem Ansatz ist, dass LOINC bislang lizenzkostenfrei genutzt werden kann und für den Großteil der Meldesachverhalte entsprechende Kodes gefunden werden konnten. LOINC stellt ein multiaxiales Begriffssystem dar, das mehrere Aspekte Achsen zuordnet (in unserem Fall relevant sind: Erreger, Material und Nachweismethode) und diese zusammen auf einem einzelnen Kode abbildet (Präkoordination). Problematisch ist daran, dass aus der Kombination von Erreger, Nachweismethode und Material eine große Zahl von Kodes resultiert. Allein für die TOP-2o der dem Landeszentrum Gesundheit NRW als Landesstelle jährlich gemeldeten Erreger wurden etwa 8 oo potentiell passende LOINC-Kodes ermittelt. Seitens der Projektpartner auf Laborseite und deren Softwarehersteller wurden deutliche Vorbehalte gegenüber einer solchen präkoordinierten Kodierung geäußert, da dort Erreger, Nachweismethode und Material in getrennten Tabellen (d.h. für jeden Aspekt gibt es eine eigene Kodeliste) abgebildet werden und ein Mapping auf die Vielzahl von präkoordinierten LOINC-Kodes als extrem aufwändig eingestuft wurde. Wenn man berücksichtigt, dass über die in den Falldefinition des RKI festgelegten statistikrelevanten Kombinationen der 55 im IfSG explizit aufgeführten Erreger hinaus, auch alle denkbaren Kombinationen von möglichen Untersuchungsmethoden und Materialien die Definition des $\mathbb{5} 7$ Abs. 1 und 2 IfSG erfüllen und damit formal meldepflichtig sind, ist die Befürchtung einer ausufernden Komplexität des zu verwendenden Value-Sets (dies wird gelegentlich auch unter dem Begriff „,kombinatorische Explosion“ diskutiert) zumindest nachvollziehbar. Dass es sich bei solchen Fällen nicht um eine rein theoretische Möglichkeit handelt, wird deutlich, wenn man bedenkt, dass auch Meldungen, die nicht unmittelbar in die gängigen Schubladen passen, durchaus ein ernstzunehmendes Ausbruchsgeschehen zum Hintergrund haben können. Ein Beispiel hierfür ist der Legionellose-Ausbruch in Warstein im Jahr 2013. Auch wenn nur die im jeweiligen Labor tatsächlich durchgeführten Untersuchungen mit LOINC abgebildet werden sollten, wäre das Heraussuchen der individuell benötigten Kodes aus Sicht der Labore zu aufwändig und im begrenzten Rahmen des Projektes nicht zu bewältigen.

Angesichts dieser Umstände wurde nach einer Lösung gesucht, wie LOINC als potentielle Alternative zu SNOMED-CT dennoch im Rahmen einer Erprobung eingesetzt werden könnte. Zunächst wurde überlegt, die Anzahl der zu mappenden Kodes dadurch zu verringern, dass nur LOINC-Kodes genutzt werden, die auf einer der relevanten Achsen (Material oder Methode) unspezifisch sind. Dies würde aber nach sich ziehen, dass hilfsweise die fehlende Achse über ein anderes Value-Set abgebildet werden müsste. Nach erster Prüfung kamen dafür nur SNOMED-CT oder ein eigenes/proprietäres Kodesystem in Frage. Im Dialog mit der Hochschule Niederrhein kristallisierte sich heraus, dass potenziell die Möglichkeit besteht, innerhalb von LOINC Achsen-Kodes, sogenannte LP-Kodes, für eine postkoordinierte Kodierung einzusetzen. Bis auf wenige Ausnahmen könnten alle auf Behördenseite im Rahmen von SurvNet@RKI [RKI, 2014] verwendeten Erreger, Methoden und Materialien mittels LP-Kodes abgebildet werden. 
B Der fachliche Hintergrund: Terminologien und Ordnungssysteme in der

Patientenversorgung, medizinischen Forschung und Gesundheitswirtschaft

\subsection{SNOMED-CT}

Als Alternative zu LOINC wird SNOMED Clinical Terms (SNOMED-CT) [IHTSDO, 2014] angesehen. Auch hierfür wurden durch das Team der Hochschule Niederrhein die entsprechenden Kodes herausgesucht. SNOMED-CT bietet die Möglichkeit, komplexe Sachverhalte in die entsprechenden Bedeutungsbestandteile (Achsen) zu zerlegen und zu kodieren, um mit einer Kombination der Einzelkodes den Sachverhalt wieder abbilden zu können (Postkoordination, vgl. auch [Ingenerf, 2007]). Dieser Ansatz bietet den Vorteil, dass die Anzahl der benötigten Kodes wesentlich geringer ist als wenn alle Bedeutungsbestandteile eines Sachverhalts in einem einzelnen Kode zusammengefasst werden. Die TOP-20-Meldesachverhalte lassen sich mittels Postkoordination durch 41 Kodes abbilden (20 Erreger, 11 Nachweismethoden, 10 Materialien). Darüber hinaus bietet SNOMED-CT als umfassende Terminologie nicht nur potentiell geeignete Kodes für Erreger, Material und Nachweismethoden, sondern darüber hinaus die Möglichkeit, die Erreger direkt mit assoziierten Erkrankungen, Lokalisation und Substanzen (z.B. Antibiotika) zu verknüpfen. Als nachteilig wird bei SNOMED-CT angesehen, dass zur Nutzung eine voraussichtlich kostenpflichtige Lizenz benötigt wird. Bei dem aktuellen NRW-Projekt wurde für die Erprobung von SNOMED-CT im Rahmen der elektronischen Labormeldung eine kostenfreie Lizenz seitens der Herausgeberorganisation IHTSDO (vgl. [IHTSDO, 2014]) gewährt. Für den Regelbetrieb wäre jedoch wahrscheinlich der Erwerb einer nationalen Lizenz in Verbindung mit dem Beitritt Deutschlands zur IHTSDO oder der Erwerb von Einzellizenzen für die jeweiligen Nutzer von SNOMED-CT erforderlich. Letzteres wäre jedoch der Akzeptanz einer elektronischen Meldung, insbesondere aus Sicht der Meldepflichtigen, eher abträglich, sofern die Lizenzkosten nicht durch finanzielle Anreize für den Melder ausgeglichen würden.

\subsection{Eigene/selbstdefinierte Kodelisten}

Eine weitere denkbare Alternative wäre die Nutzung von eigenen Kodelisten. Hier könnte man für die zu übermittelnden Meldesachverhalte eigenen Begriffe/Values definieren oder bestehende proprietäre Kodelisten (z.B. die innerhalb von SurvNet@RKI verwendeten Kodes) verwenden. Vorteilhaft wäre, dass man keine Probleme damit hätte, die benötigten Bedeutungen auf ein "fremdes" Vokabular abzubilden. Allerdings wäre die langfristige Pflege und Bereitstellung eines eigenen Kodesystems mit einem erheblichen Aufwand verbunden. Auch wäre ein solches Kodesystem inkompatibel mit anderen im Gesundheitswesen eingesetzten Klassifikationen oder Terminologien. In der Folge stünde dem Aufwand für die Implementierung eines solchen Kodesystems durch die Softwarehersteller nur ein sehr begrenzter Nutzen gegenüber, da es sich kaum für andere Zwecke wiederverwenden ließe. Aus diesem Grund erscheint dieser Ansatz weniger vorteilhaft als die zuvor betrachteten Lösungs- 
ansätze. Daher eigenen sich selbsterstellte Kodesysteme primär als Behelfslösungen, falls es nicht anders möglich wäre, die benötigten Begriffe und Bedeutungen abzubilden.

\subsection{Fazit und Ausblick}

Nach den bisher im Rahmen der Aktivitäten in NRW gemachten Erfahrungen deutet vieles darauf hin, dass die Verwendung einer standardisierten Terminologie für Zwecke des Meldewesens praktikabel ist. Für die Arztmeldung bietet es sich an, auf die bereits in den Primärsystemen vorhandene ICD-10 zurückzugreifen. Auch wenn es sowohl bei LOINC als auch bei SNOMED-CT nicht vollständig gelungen ist, für alle in Deutschland in diesem Kontext gebräuchlichen Konzepte (Erreger, Nachweismethoden und Materialien) passende Kodes mit der notwendigen semantischen Äquivalenz zu finden, so ist dies jedoch für die überwiegende Anzahl der benötigten Konzepte möglich. Hinsichtlich dieser Lücken gibt es die Möglichkeit, entweder die fehlenden Kodes bei der jeweiligen Herausgeberorganisation - d.h. beim Regenstrief Institute, bzw. der IHTSDO - zu beantragen oder übergangsweise eigene Kodes in das benötigte Value-Set aufzunehmen. Hier erhofft man sich aus der anstehenden pilothaften Implementierung und dem für die zweite Jahreshälfte 2014 angestrebten Erprobungsbetrieb weitergehende Erkenntnisse. Seitens der in NRW eingebundenen Labore und Softwarehersteller wurde eine deutliche Präferenz für eine postkoordinierte Kodierung geäußert, da hier der Implementierungsaufwand erheblich geringer eingeschätzt wird. Für diesen Zweck bieten sich SNOMED-CT oder alternativ die LOINC-LP-Kodes an. Sollte man auf nationaler Ebene auf den Einsatz von präkoordinierten LOINC-Kodes setzen, wäre es in jedem Fall erforderlich, die Labore und deren Softwarehersteller bei der Überführung der laboreigenen Kodesysteme in LOINC zu unterstützen. Hier wäre erwägenswert, ob man die notwendige semantische Normalisierung erst beim Empfänger vornimmt. Im Zusammenhang mit den „Meaningful Use Regulations “ [HealthIT.gov, 2014] wird in den USA in einigen Krankenhäusern bereits ein automatisiertes Mapping durchgeführt, das mittels einer softwaregestützten semantischen Analyse bis zu 70 Prozent der Texte von Volltext-Arztbriefen in standardisierte Vokabulare überführen kann [3M, 2013]. Da die Vokabulare im Laborbereich im Zeitverlauf relativ wenigen Änderungen unterliegen, könnte hier wahrscheinlich eine deutlich höhere Erkennungsrate erreicht werden.

Eine wichtige Erkenntnis ist bereits jetzt, dass es zwingend angeraten erscheint, bei der Einführung eines solchen Systems diejenigen, die es hinterher implementieren und in der Praxis nutzen sollen (d.h. im jeweiligen Fall die Ärzte, Krankenhäuser und Labore sowie deren Softwarehersteller) frühzeitig bei der Konzeption einzubinden. 
B Der fachliche Hintergrund: Terminologien und Ordnungssysteme in der

Patientenversorgung, medizinischen Forschung und Gesundheitswirtschaft

\section{Referenzen}

[3M, 2013] 3M Health Information Systems (2013): Innovating the Language of Health - Meaningful Use and the $3 \mathrm{M}$ Healthcare Data Dictionary.

[BVA, 2014] BVA. Bundesverwaltungsamt - Verbindungsnetz DOI. www.bva.bund.de/DE/Organisation/ Abteilungen/Abteilung_BIT/Leistungen/IT_Produkte/VerbindungsnetzDOI/verbindungsnetzdoi_node. htm/ Letzter Zugang: 2014-08-12.

[D2D, D2D. Die Telematik-Plattform der Kassenärztlichen Vereinigungen. http://www.d2d.de/Letzter Zugang: 2014-08-12.

[DIMDI, 2014] DIMDI. ICD-10-GM. http://www.dimdi.de/static/de/klassi/icd-10-gm/index.htm Letzter Zugang: 2014-08-12.

[Fraunhofer-Wiki,2014] Fraunhofer-Wiki.DEMISAP2.publicwiki-01.fraunhofer.de/Planungsstudie_Interoperabilitaet/ index.php/DEMIS_AP2 Letzter Zugang: 2014-08-12.

[gematik, 2014a] gematik. Fachkonzept Mehrwertanwendungen - Mehrwertkommunikation Leistungser-

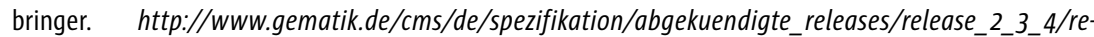
lease_2_3_4_fachanwendungen/fachanwendungen/release_2_3_4_fachkonzept_mwk_le.jsp Letzter Zugang: 2014-08-12.

[gematik,2014b]gematik.Telematikinfrastruktur.http://www.gematik.de/cms/de/egk_2/telematikinfrastruktur/ telematikinfrastruktur_1.jsp Letzter Zugang: 2014-08-12.

[HealthIT.gov, 2014] HealthIT.gov. Health IT Regulations - Meaningful Use Regulations. http://www.healthit. gov/policy-researchers-implementers/meaningful-use-regulations Letzter Zugang: 2014-08-12.

[HL7, 2007-2014a] HL7. Arztbrief 2006 - Implementierungsleitfaden. http://wiki.hl7.de/index.php/IG:Arztbrief _2006 Letzter Zugang: 2014-08-14.

[HL7, 2007-2014b] HL7. Meldewesen und Infektionsschutz (Projekt).http://wiki.hl7.de/index.php/Meldewesen _und_Infektionsschutz_\%28Projekt\%29 Letzter Zugang: 2014-08-12.

[IHTSDO, 2014] IHTSDO. SNOMED CT. http://www.IHTSDO.org/SNOMED-ct Letzter Zugang: 2014-08-12.

[Ingenerf, J., 2007] Ingenerf, J. (2007): Die Referenzterminologie SNOMED CT: von theoretischen Betrachtungen bis zur praktischen Implementierung. MMI.

[KBV, 2014a] KBV. Kassenärztliche Bundesvereinigung - Sicheres Netz. http://www.kbv.de/html/sicheres netz.php Letzter Zugang: 2014-08-12.

[KBV, 2014b] KBV. Zertifizierung. www.kbv.de/html/zertifizierung.php Letzter Zugang: 2014-08-12.

[KoSIT, 2014] KoSIT. XÖV - OSCI Transport. http://www.xoev.de/detail.php?gsid=bremen83.c.3355.de Letzter Zugang: 2014-08-12.

[KV-Telematik,KV-Telematik.KV-Connect.http://www.kv-telematik.de/aerzte-und-psychotherapeuten/kv-connect/ Letzter Zugang: 2014-08-12.

[LZG.NRW, 2012] Landeszentrum Gesundheit Nordrhein-Westfalen (2012): Die pandemische Influenza (H1N1) 2009 in Nordrhein-Westfalen. Rückblicke - Einblicke - Ausblicke.

[Mette, A. et al., 2011] Mette, A. et al. (2011): Untererfassung von Masern: Eine Evaluation basierend auf Daten aus Nordrhein-Westfalen. Dtsch Arztebl International 108 (12): S. 191-196.

[Regenstrief-Institute, 1994-2014] Regenstrief-Institute. LOINC ${ }^{\circledR}$ A universal code system for tests, measurements, and observations. $h$ ttp://LOINC.org/Letzter Zugang: 2014-08-12.

[RKI,2013]RKI.RobertKochInstitut-Falldefinitionen.http://www.rki.de/DE/Content/Infekt/IfSG/Falldefinition/ falldefinition_node.html Letzter Zugang: 2014-08-12.

[RKI, 2014] RKI. Software - SurvNet@RKI. http://www.rki.de/DE/Content/Infekt/IfSG/Software/software inhalt.html Letzter Zugang: 2014-08-12. 